\title{
Ubiquitination modification: critical regulation of IRF family stability and activity
}

\author{
Bao-qin $\mathrm{Liu}^{1,2}$, Jin $\mathrm{Jin}^{2,3} \&$ Yi-yuan $\mathrm{Li}^{1,2^{*}}$ \\ ${ }^{1}$ Key Laboratory for Developmental Genes and Human Disease, Ministry of Education, Institute of Life Sciences, Jiangsu Province High-Tech \\ Key Laboratory for Bio-Medical Research, Southeast University, Nanjing 210096, China; \\ ${ }^{2}$ MOE Laboratory of Biosystem Homeostasis and Protection, and Life Sciences Institute, Zhejiang University, Hangzhou 310058, China; \\ ${ }^{3}$ Sir Run Run Shaw Hospital, College of Medicine Zhejiang University, Hangzhou 310016, China
}

Received July 24, 2020; accepted August 11, 2020; published online October 30, 2020

\begin{abstract}
Interferon regulatory factors (IRFs) play pivotal and critical roles in innate and adaptive immune responses; thus, precise and stringent regulation of the stability and activation of IRFs in physiological processes is necessary. The stability and activities of IRFs are directly or indirectly targeted by endogenous and exogenous proteins in an ubiquitin-dependent manner. However, few reviews have summarized how host E3 ligases/DUBs or viral proteins regulate IRF stability and activity. Additionally, with recent technological developments, details about the ubiquitination of IRFs have been continuously revealed. As knowledge of how these proteins function and interact with IRFs may facilitate a better understanding of the regulation of IRFs in immune responses or other biological processes, we summarized current studies on the direct ubiquitination of IRFs, with an emphasis on how these proteins interact with IRFs and affect their activities, which may provide exciting targets for drug development by regulating the functions of specific E3 ligases.
\end{abstract}

IRFs, ubiquitination, E3 ubiquitin ligase, viral proteins, activation, degradation

Citation: Liu, B., Jin, J., and Li, Y. (2021). Ubiquitination modification: critical regulation of IRF family stability and activity. Sci China Life Sci 64, 957-965. https://doi.org/10.1007/s11427-020-1796-0

\section{Introduction}

The interferon regulatory factor (IRF) family of transcription factors consists of nine members (IRF1-9) in mammalian cells (Tamura et al., 2008). All IRFs share a well-conserved N-terminal DBD (DNA-binding domain), $\sim 120$ amino acids of which form a helix-loop-helix motif for the recognition of a DNA sequence. The C-terminal regions of IRFs have low sequence homology and function as association domains by which IRFs interact with other family members or transcription factors (Figure 1) (Jefferies, 2019; Tamura et al., 2008). IRFs play important roles in immune defense, stress responses, reproduction, development, and carcinogenesis,

*Corresponding author (email: 103200067@seu.edu.cn) which account for many aspects of innate and adaptive immune responses (Nehyba et al., 2009). IRF1 and IRF2, the first IRF family members identified, are well known for their antagonistic regulation of oncogenesis (Harada et al., 1993). Moreover, IRF1 and IRF2 have also been demonstrated to regulate both lymphoid and myeloid cell development and differentiation. Three other family members, IRF4, IRF5 and IRF8, are also involved in regulating immune cell development and phonotype (Jefferies, 2019; Savitsky et al., 2010). IRF3, IRF7, and IRF5 are best known for their critical role as transcription factors promoting IFN- $\alpha / \beta$ expression in type I IFN-dependent innate immune responses (Yanai et al., 2012). IRF9 is a regulator of interferon-driven gene expression that acts as a critical link between type I IFNs and the p53 pathway (Takaoka et al., 2003). Unlike other IRFs, 
DBD

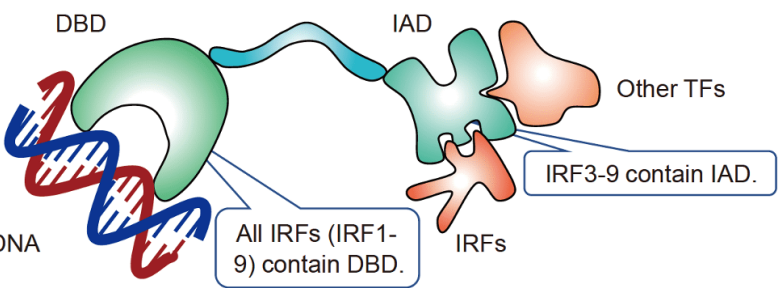

Figure 1 Schematic illustration of IRF family members. IRF family members share a well-conserved N-terminal DBD (DNA-binding domain), $\sim 120$ amino acids of which form a helix-loop-helix motif that recognizes a DNA sequence. Except for IRF1 and IRF2, the C-terminal regions of all IRF family members contain an IAD (IRF association domain) by which IRFs interact with other family members or transcription factors. TFs: transcription factors.

IRF6 has essential functions in the normal development and differentiation of the epidermis instead of participating in innate immunity (Richardson et al., 2006).

Due to the pivotal and critical roles of IRFs, the balance between the activation and destruction of IRFs needs to be constantly maintained to avoid adverse overreaction. To orchestrate an appropriate immune response, the immune system is strictly regulated. Various posttranslational modifications (PTMs), such as phosphorylation, ubiquitination, acetylation and other unconventional PTMs, have evolved as critical regulators of the immune system, and all PTMs are important in regulating the activity, stability and folding of IRFs (Deribe et al., 2010). The most extensively investigated type of PTM in innate immunity is phosphorylation, which is inversely regulated by kinases and phosphatases (Karin and Ben-Neriah, 2000; Liu et al., 2016). Similar to phosphorylation, ubiquitination is one of the most important regulatory mechanisms in the immune system, and ubiquitination can also be reversibly regulated by E3 ubiquitin ligases and DUBs (deubiquitinating enzymes) (Ning et al., 2011).

Ubiquitination is a common modification for protein activation or deactivation, and IRFs are stringently regulated by ubiquitination in many respects. Ubiquitination can directly or indirectly target IRFs and positively or negatively regulate the stability, activation and transcriptional activity of IRFs (Ning et al., 2011). Through the ubiquitin pathway, IRFs can improve the antiviral response by ubiquitin-mediated degradation or activation. This review summarized current research on the direct ubiquitination of IRFs, with an emphasis on several host proteins and viral proteins found to target IRFs for ubiquitination or deubiquitination. Knowledge of how these proteins function and interact with IRFs may provide a better understanding of the regulation of IRFs in immune responses or other biological processes and exciting targets for the development of drugs aimed at regulating the functions of specific E3 ligases or DUBs.

\section{Brief introduction to ubiquitination}

Ubiquitination is a type of reversible cellular protein PTM. Ubiquitin, the core component of ubiquitination, is covalently attached to one or more lysine residues in cellular proteins through an enzymatic cascade involving three classes of enzymes termed ubiquitin-activating enzymes (E1), ubiquitin-conjugating enzymes (E2), and ubiquitin protein ligases (E3) (Heaton et al., 2016; Mukhopadhyay and Riezman, 2007). The human proteome contains two E1 enzymes, approximately 50 E2 enzymes, 600 E3 ligases, and 100 DUBs (Bhoj and Chen, 2009). Among these, E3 ubiquitin ligases largely dictate substrate specificity. E3 ligases can be generally divided into three subgroups: the HECT (homology to E6-associated protein carboxyl terminus) domain E3 ligases, RING (really interesting new gene)-type E3 ligases, and RBR (RING-between-RING) E3 ligases (Dove and Klevit, 2017). The ubiquitinating modification can be reversed by DUBs. DUBs are essentially proteases that cleave the peptide or isopeptide bond between ubiquitin and its substrate protein (Nijman et al., 2005). There are two classes of DUBs: cysteine proteases, which can be further divided into four different classes (UCHs (ubiquitin-Cterminal hydrolases), USPs (ubiquitin-specific proteases), MJDs (Machado-Joseph Disease protein domain proteases), and OTUs (ovarian tumor proteases), and metalloproteases, which contain only JAMMs (JAMM motif proteases) (Chen and Sun, 2009).

Ubiquitin itself contains seven lysine residues and one Nterminal methionine residue (K6, K11, K27, K29, K33, K48, $\mathrm{K} 63$ and M1), all of which can be further conjugated by another ubiquitin to form ubiquitin chains with different linkages (Ikeda and Dikic, 2008). Various types of ubiquitin chains carry a range of molecular signals and function in a variety of cellular processes, including membrane trafficking, protein kinase activation, DNA repair, and cell signaling (Chen and Sun, 2009; Welchman et al., 2005). K48-linked polyubiquitination is a well-studied type of ubiquitination by which proteins are targeted for proteasomal degradation through the 26S proteasome (Zheng and Shabek, 2017). Over the last two decades, mounting evidence has identified atypical linkages that mediate less frequent proteasomal degradation, such as K11- and K29-linked chains (Komander and Rape, 2012). K27-linked ubiquitination is required for proper activation of the DNA damage response, and K27linked polyubiquitination of STING facilitates TBK1 recruitment and activation (Gatti et al., 2015; Wang et al., 2014). M1-linked linear polyubiquitination is involved in the activation of NF- $\mathrm{kB}$ signaling (Tokunaga et al., 2009). The activation of $\mathrm{T}$ cells is negatively regulated by K33-linked polyubiquitination (Huang et al., 2010; Yang et al., 2015). Only a few studies have demonstrated that K6-linked ubiquitination may be involved in the DNA damage response 
and the maintenance of mitochondrial stability (Jin et al., 2010; Wu-Baer et al., 2003). Furthermore, K63-linked polyubiquitination and monoubiquitination, the second most common type of ubiquitin linkage, mediate proteasome-independent effects, which play an important role in activating many components of different signaling pathways and participate in many biological processes (Ning et al., 2011).

\section{Cellular E3/DUB-mediated regulation of IRFs}

Because of the critical functions of IRF family members in regulating the activation of IFN promoters and downstream signaling in cell differentiation and tumor suppression, it is of vital importance that their activities are maintained in a state that is not overly stimulated. Ubiquitin-mediated proteasomal degradation of IRF family members is an effective mechanism to limit IRF activity, and ubiquitin-mediated activation of IRFs is also essential for IRFs to better regulate related biological processes. Many studies, especially on IRF3 and IRF7, have identified some endogenous proteins that function as E3 ubiquitin ligases and catalyze IRF ubi- quitination in a proteasome-dependent pathway or a nonproteolytic pathway (Figure 2) (Higgs and Jefferies, 2008; Tsuchida et al., 2009).

\section{IRF3/7}

\section{Ubiquitin-mediated degradation of IRF3/7}

IRF3 and IRF7 are best known for their critical role as master regulators promoting type I IFN expression. IRF3 is constitutively expressed, and IRF7 is an IFN-stimulated gene (ISG) whose expression can be stimulated by interferon (Higgs and Jefferies, 2008; Lazear et al., 2013). Following viral infection, the host produces interferon against pathogen infections, and interferon activates the JAK-STAT signaling pathway to induce the transcription of ISGs (Schneider et al., 2014), which can positively induce the expression of IRF7 and drive more interferon. As IRF3 and IRF7 are essential switches that turn off and limit type I IFN production to avoid overreaction, ubiquitin-mediated degradation of IRF3 and IRF7 has been well described in many studies.

As a member of the TRIM family, TRIM21 (also called Ro52) was initially characterized as an autoantigen in pa-

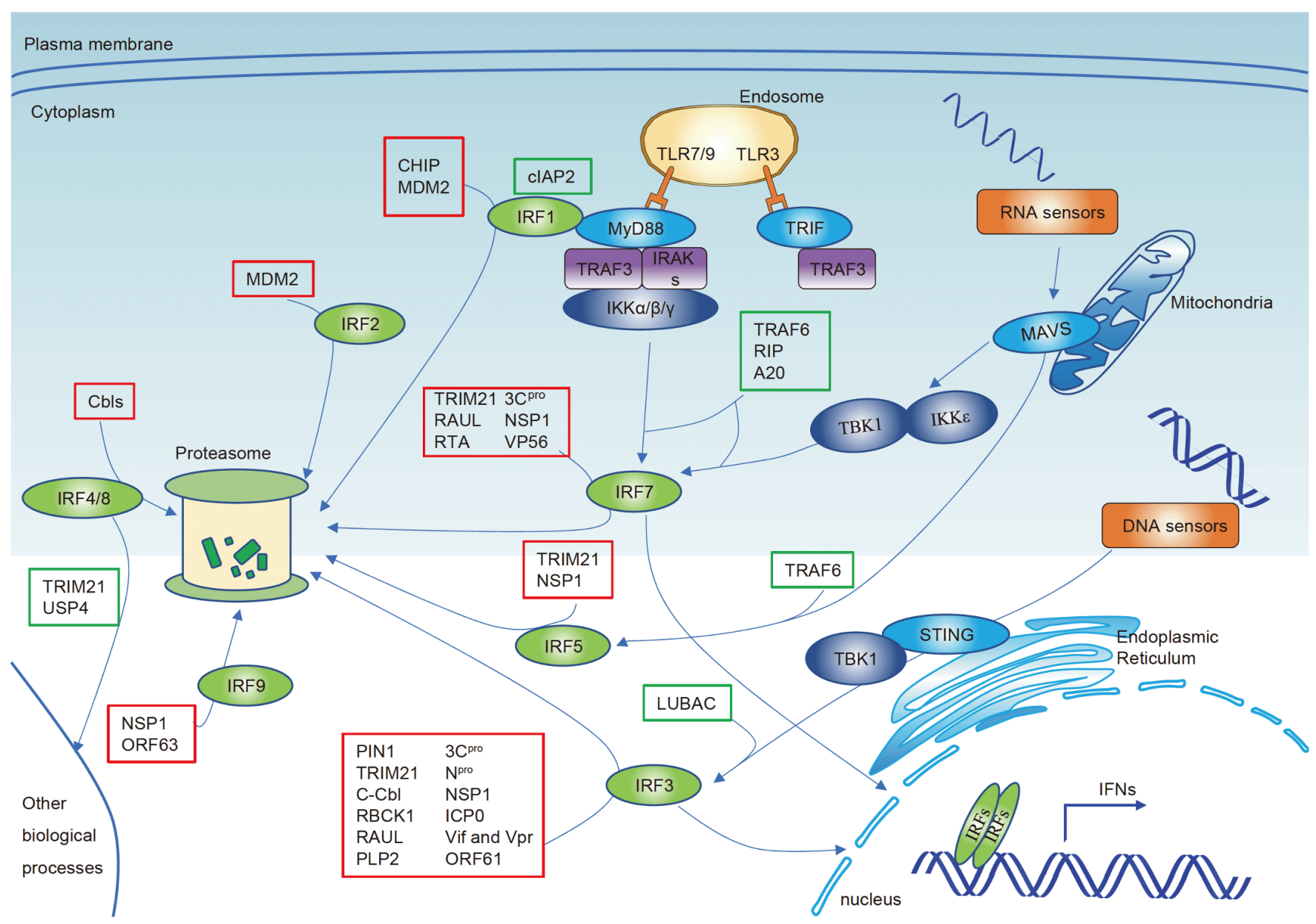

Figure 2 Ubiquitin-mediated regulation of IRF signaling. Host E3 ligases and viral proteins that regulate IRF family members are highlighted in boxes. Green boxes indicate positive regulators, and red boxes indicate negative regulators. 
tients with autoimmune diseases, including Sjögren's syndrome and systemic lupus erythematosus (Rhodes et al., 2002). Upon RNA virus infection, TRIM 21 is significantly induced and functions as an E3 ubiquitin ligase, enabling it to play important physiological roles in many relevant biological processes. IRF3, IRF5, IRF7 and IRF8 can act as substrates for TRIM21-mediated ubiquitination. Via its Cterminal SPRY domain, TRIM21 negatively regulates the stability of IRF3 by mediating IRF3 polyubiquitination and proteasomal degradation (Higgs et al., 2008). However, some studies have shown that TRIM21 sustains IRF3 activation during the antiviral response at the early phase of infection (Yang et al., 2009). PIN1 (peptidyl-prolyl isomerase) recognizes phosphorylated IRF3 at the S339-P340 motif and finally targets IRF3 for degradation through polyubiquitination (Chen et al., 2018; Saitoh et al., 2006). TRIM21 can interfere with the interaction between PIN1 and IRF3, which prevents IRF3 ubiquitination and degradation (Yang et al., 2009). In addition, studies have identified a novel role for tyrosine phosphorylation in regulating the activity of TRIM21. Upon TLR3 and TLR4 stimulation, TRIM21 is tyrosine phosphorylated, which can simultaneously positively regulate its interaction with IRF3 and enhance TRIM21 activity (Stacey et al., 2012). This may explain the complicated role of TRIM21 in IRF3 regulation.

In the case of IRF7, TRIM21-mediated ubiquitination promotes the degradation of IRF7 following TLR7 and TLR9 stimulation (Higgs et al., 2010). The interaction of TRIM21 with the apoptotic protein Fas-associated death domain (FADD) can enhance the ubiquitin ligase activity of TRIM21, and both TRIM21 and FADD cooperatively interact with IRF7 and contribute to TRIM21-mediated ubiquitination of IRF7 (Young et al., 2011).

Three other cellular E3 ligases have been identified, c-Cbl, RBCK1 and RAUL (RTA-associated ubiquitin ligase), all of which target and promote K48-linked polyubiquitinationdependent proteasomal degradation of IRF3. c-Cbl, a member of the Cbl (Casitas B-lineage lymphoma) family, negatively regulates IRF3 protein stability by interacting with the C-terminal domain of IRF3 via its TKB (tyrosine kinase binding) domain and promotes K48-linked polyubiquitination-dependent proteasomal degradation of IRF3 (Zhao et al., 2016). After induction by viral infection, the E3 ubiquitin ligase RNF54, also called RBCK1 (RBCC protein interacting with $\mathrm{PKC} 1$ ), specifically catalyzes the K48linked polyubiquitination of IRF3, which mediates the degradation of IRF3. This process is an important negative feedback mechanism for the termination of IRF3-dependent antiviral responses (Zhang et al., 2008). Additionally, RBCK1 plays a broader role in the suppression of host antiviral responses because it suppresses NF- $\mathrm{KB}$ activation by negatively regulating TNF and IL-1 (Tian et al., 2007). The HECT E3 ligase RAUL directly catalyzes K48-linked polyubiquitination of both IRF3 and IRF7, followed by their proteasome-dependent degradation. The viral E3 RAT encoded by KSHV (Kaposi's sarcoma-associated virus) also cooperates with the host E3 ligase RAUL to increase proteolysis of both IRF7 and IRF3, which is more effective against the host antiviral response (Yu and Hayward, 2010).

IRF3/7 activity is affected by ubiquitination or deubiquitination

Not only is ubiquitination involved in protein degradation but also the proteasome-independent functions of nondegradative ubiquitination are required to promote the activation of IRF3 and IRF7 (Higgs and Jefferies, 2008). In addition, IRF activity is positively or negatively regulated by some DUBs that regulate IRFs by removing the ubiquitin chain from ubiquitinated IRFs (Figure 2).

Linear ubiquitin chain assembly complex (LUBAC) is an ubiquitin ligase complex composed of SHARPIN, HOIL-IL and HOIP that generates linear polyubiquitin chains (Tokunaga and Iwai, 2012). The activation of IRF3 in the RIPA (RLR-induced IRF3-mediated pathway of apoptosis) requires linear polyubiquitination of IRF3 at two specific lysine residues mediated by LUBAC, which triggers its interaction with the proapoptotic protein Bax to induce mitochondrial activation and apoptotic cell death (Chattopadhyay et al., 2016; Chattopadhyay and Sen, 2017).

IRF7 activation by Epstein-Barr virus LMP1 (latent membrane protein 1) and IKK $\varepsilon$ is enhanced following K63linked ubiquitination by TRAF6. TRAF6 ubiquitinates IRF7 at multiple sites, but this ubiquitination is independent of the C-terminal functional phosphorylation sites of IRF7, which provides evidence that the regulatory ubiquitination of IRF7 is a prerequisite for its phosphorylation (Ning et al., 2008). In addition, receptor-interacting protein (RIP) contributes to LMP1-promoted ubiquitination of IRF7 and is required for full activation of IRF7 by LMP1, suggesting that RIP serves as a general activator of IRF7 (Huye et al., 2007). The antiapoptotic factor A20, which possesses both DUB activity in its $\mathrm{N}$-terminal domain and $\mathrm{E} 3$ ligase activity in its $\mathrm{C}$-terminal domain, is also induced by LMP1. The A20 N-terminal DUB domain interacts with and deubiquitinates IRF7, decreasing the K63-linked ubiquitination of IRF7 (Ning and Pagano, 2010).

\section{IRF1/2}

The regulation of IRF1 and IRF2 is considerably less well understood than that of IRF3 and IRF7. IRF1 and IRF2 are well known for their antagonistic regulation of oncogenesis. As a tumor suppressor protein, IRF1 exhibits a short half-life and turns over rapidly in response to changes in the cellular environment (Pion et al., 2009). The IRF1 protein level is differentially regulated by CHIP (C terminus of the Hsc 
(heat-shock cognate) 70-interacting protein). CHIP, a protein quality control E3 ligase, is regarded as a link between protein folding and protein degradation pathways through its selective ubiquitination of misfolded proteins via collaboration with molecular chaperones (Murata et al., 2001). In unstressed cells, CHIP appears to chaperone IRF1 and positively affect its protein levels. However, under certain stress conditions, CHIP binds a central intrinsically disordered domain (Mf2 domain) of IRF-1 and mediates its ubiquitination (Narayan et al., 2011). The structured DBD of IRF1 acts as an ubiquitin acceptor site of CHIP binding and ubiquitination (Landré et al., 2013). Importantly, the interaction between IRF1 and CHIP was enhanced by trichostatin A (TSA) treatment, which facilitated the degradation of IRF1 and was shown to be important in the TSA-mediated inhibitory effect on IFN- $\gamma$ induction mediated by TRIM 22 and other IRF-1-dependent IFN-stimulated genes (Gao et al., 2013).

In addition, MDM2 functions as an Mf2 domain ligase, interacts with the IRF1 DBD and mediates the ubiquitination of IRF1 (Landré et al., 2013). MDM2 (murine double minute 2 , also known as human double minute 2 protein (HDM2)) is known for its role in targeting p53 for ubiquitin-mediated proteasomal degradation; it also interacts with IRF2 and mediates its ubiquitination (Moll and Petrenko, 2003). The interaction between IRF2 and MDM2 requires both the hydrophobic pocket and acidic domain of MDM2, and its dual binding sites enable E3-ubiquitin ligase-substrate interactions with IRF2, which may attenuate the function of IRF2 as a transcriptional repressor (Pettersson et al., 2009).

In addition, the activation of IRF1 requires K63-linked polyubiquitination by the apoptosis inhibitor cIAP2 during IL-1 signaling. The E3 ubiquitin ligase activity of cIAP2 can be enhanced by the bioactive sphingolipid mediator S1P, and both cIAP2 and S1P form a complex with IRF1, which is essential for IL-1-induced production of the chemokines CXCL10 and CCL5 (Harikumar et al., 2014).

\section{IRF $4 / 5 / 8$}

IRF4, IRF5 and IRF8, the stability and activity of which are also critically regulated by ubiquitination, play important roles in regulating the development and differentiation of both lymphoid and myeloid cells. As described above, the other two Cbl family members in mammals, Cbl-b and Cblc/Cbl-3, are also involved in negatively regulating the stability of IRFs (Swaminathan and Tsygankov, 2006). All Cbl proteins have a conserved N-terminal region that encompasses a TKB domain, RING finger domain and a linker region between them. These domains enable $\mathrm{Cbl}$ proteins to function as E3 ligases (Swaminathan and Tsygankov, 2006; Thien and Langdon, 2005). Cbl proteins play a critical role in antibody affinity maturation in GC (germinal center) B cells by promoting IRF4 ubiquitination and degradation ( $\mathrm{Li}$ et al., 2018). Likewise, the stability of IRF8 is downregulated by Cbl-mediated ubiquitination, and the C-terminal domain of IRF-8 is necessary for ubiquitination (Xiong et al., 2005). IRF5 contains polymorphisms due to its alternative splicing and the insertion or deletion of a 30-nucleotide sequence in IRF5 exon 6. As mentioned, IRF5 can be a substrate of TRIM21, and various IRF5 isoforms can directly interact with TRIM21 upon TLR7 stimulation. IRF5-V1 and IRF5V5 are targeted for TRIM21-mediated degradation, whereas IRF5-V2 and IRF5-V3 are resistant to TRIM21-mediated degradation, which is of great importance in regulating the stability and activity of IRF5 (Lazzari et al., 2014).

Proteasome-independent ubiquitination is also involved in regulating these three IRFs. Upon IFN- $\gamma$ and TLR stimulation in murine macrophages, TRIM21 was found to interact with IRF8 and ubiquitinate IRF8 in the nondegradative pathway, which positively contributed to the expression of IL-12 p40 (Kong et al., 2007). IRF8 protein levels in regulatory T cells are also stabilized by USP4 (ubiquitin-specific protease 4), and USP4 physically interacts with IRF8 and functions via K48-linked deubiquitinase activity (Lin et al., 2017). USP4 also stabilizes IRF4 protein levels by interacting with and deubiquitinating IRF4, which promotes the function of IRF4 in facilitating IL-4 expression in T helper type 2 cells (Guo et al., 2017). Similarly, upon TLR7 or TLR9 stimulation, TRAF6 interacts with IRF5 and promotes K63-linked ubiquitination of IRF5, which is important for IRF5 nuclear translocation and target gene regulation (Balkhi et al., 2008).

\section{Exogenous protein-mediated regulation of IRFs}

There is mounting evidence that IRF activity is also downregulated by the ubiquitination of viral proteins. Viruses have evolved a multitude of strategies to disturb the innate immune system. Viruses facilitate their own replication, and their persistence largely depends on their ability to exploit the ubiquitin system; viruses can encode their own E3 ligases or deubiquitinases or redirect host ubiquitin enzymes (Marsili et al., 2016; Randow and Lehner, 2009). IRFs are generally an attractive ubiquitination target for the virusmediated subversion of host antiviral responses (Figure 2).

As the dominant regulators of type I IFN production following viral infection, the ability of IRF3 and IRF7 to impair viral replication makes them the most important targets for many viruses. The protein expression and phosphorylation of IRF3 and IRF7 were shown to be inhibited after SVV (Seneca Valley virus) infection, and SVV $3 C^{\text {pro }}$ interacted with IRF3 or IRF7 in PK-15 cells and contributed to the degradation of IRF3 and IRF7 through its protease activity (Xue et al., 2018a). Furthermore, the cysteine and histidine 
residues of SVV $3 C^{\text {pro }}$ endow it with deubiquitinating activity, which can inhibit both the K48-linked polyubiquitination of TBK1 (TANK-binding kinase 1) and the K63-linked polyubiquitination of RIG-1 (retinoic acid-inducible gene I) and TRAF3 (TNF receptor-associated factor 3). Interestingly, $3 \mathrm{C}^{\text {pro }}$ partly stabilizes $\mathrm{TBK} 1$ by its DUB activity, but as a whole, the expression level of TBK1 is still reduced after SVV infection. Therefore, via its DUB activity, SVV $3 C^{\text {pro }}$ suppresses the RIG-I- and TBK1-induced expression of IFN- $\beta$ (Xue et al., 2018b).

The ICP0 (infected cell protein 0) protein encoded by several herpesviruses also functions as an E3 ligase and induces K48-linked ubiquitination and proteolysis of IRF3 and IRF7. The RING finger domain of ICP0 is essential for its E3 ubiquitin ligase activity. However, some studies demonstrated that ICP0 encoded by BICP0 (bovine herpesvirus 1) reduced the protein levels of IRF3 but not IRF7 and directly or indirectly targeted IRF3 for proteasome-dependent degradation (Saira et al., 2007; Saira et al., 2009). Moreover, $\mathrm{BICP0}$ was also found to promote the K48-linked ubiquitination of TRAF6 and mediate its degradation through a proteasome-dependent pathway, influencing the activation of IRF7 via K63-linked ubiquitination by TRAF6 (Cao et al., 2019). Studies have identified full-length rotavirus NSP1 (nonstructural protein 1) as a viral IFN antagonist that induces the proteasome-mediated degradation of IRF3 (Barro and Patton, 2005). As NSP1 specifically targets the Cterminal regions of IRFs (except IRF1 and IRF2), which carry an IRF association domain that mediates homo or heterodimeric IRF interactions, NSP1 also mediates the degradation of IRF5, IRF7 and IRF9 (Arnold, 2016; Barro and Patton, 2007).

During the early steps of HIV-1 infection, the HIV-1 accessory proteins Vif and Vpr decrease the relative levels of IRF3 due to ubiquitin-associated proteasome degradation without activating IRF3. The N-terminal lysine residues of IRF3 are important for Vif- and Vpr-mediated degradation (Doehle et al., 2009; Okumura et al., 2008). However, the degradation of IRF3 by another accessory protein, Vpu, remains controversial (Doehle et al., 2012; Langer et al., 2019). The cellular E3 ligase HDM2 is exploited by the transcriptional activator Tat of HIV-1, which accelerates IRF1 proteasome-mediated degradation (Remoli et al., 2016). In addition, inactivated IRF3 can interact with the viral protein $\mathrm{N}^{\mathrm{pro}}$ (N-terminal protease), a small papain-like cysteine protease. $\mathrm{N}^{\text {pro }}$ interacts with and induces the degradation of IRF3 upon infection with the pestiviruses classical swine fever virus (CSFV) and bovine viral diarrhea virus (BVDV), which causes a marked loss of IRF3 (Bauhofer et al., 2007; Chen et al., 2007). ORF61 and PLP2 can recognize and interfere with activated IRF3. ORF61, an immediate-early protein of varicella-zoster virus $(\mathrm{VZV})$, inhibits the Sendai virus-mediated activation of IFN- $\beta$ by directly interacting with activated IRF3, leading to the degradation of IRF3 through an ubiquitin-proteasome pathway (Zhu et al., 2011). Papain-like protease domain 2 (PLP2), a catalytic domain of nsp3 (nonstructural protein 3) from MHV-A59 (mouse hepatitis virus A59), contains a conserved DUB motif. PLP2 binds IRF3 and causes its deubiquitination to prevent the nuclear localization of IRF3. Moreover, the DUB activity of PLP2 can cleave both K48- and K63-linked polyubiquitin chains from IRF3, which may be why coronaviruses can escape host innate antiviral responses (Tsuchida et al., 2009; Zheng et al., 2008).

As mentioned before, the KSHV-encoded immediate-early protein RTA (RNA transcriptional activator, also known as ORF50) has evolved the ability to redirect the host E3 ligase RAUL to strengthen the proteolysis of both IRF7 and IRF3. The effect of KSHV against the host antiviral response is also attributed to the E3 ligase activity of RTA, which targets IRF7 for proteasome-dependent degradation. A cystine- and histidine-rich N-terminal domain of RTA is critical for its E3 ligase activity (Yu et al., 2005). GCRV (grass carp reovirus) VP56 blocks the host IFN response and facilitates viral replication by inducing K48-linked ubiquitination and degradation of phosphorylated IRF7 upon GCRV infection (Zhang et al., 2020). In addition to NSP1, IRF9 is targeted by the immediate-early protein ORF63 encoded by VZV and simian varicella virus, which blocks JAK-STAT signaling by degrading IRF9 in a proteasome-dependent manner (Verweij et al., 2015).

\section{Conclusions and perspectives}

The important roles played by IRFs in specific type I IFN induction and how IRFs are activated and regulated by viral infection and PTMs have been discussed in many recent reviews. This review summarizes current research on the direct ubiquitination of IRFs and introduces and classifies identified cellular E3 ligases/DUBs and viral proteins by substrate specificity, interaction and effect on IRF stability or activity (Table 1). However, research on how ubiquitination regulates IRFs is still being updated with more in-depth studies on the mechanism by which IRFs shape innate and adaptive immune responses, cell differentiation, tumor suppression and other biological processes. Many questions remain unanswered and require explanation: the ubiquitin acceptor sites of some IRFs that undergo E3 ligase-mediated ubiquitination remain undefined, the interactions between E3 ligases or viral proteins and IRFs and their functions should be better characterized, and how viruses exploit the ubiquitin system to interact with and affect the host immune system should be further elaborated. Thus, further identification of these sites can fully explain the role of ubiquitin in regulating IRFs, and interfering viral protein-IRF interaction interfaces 
Table 1 Summary of host and viral proteins that regulate the stability or activity of IRFs by degradation or by promoting activation

\begin{tabular}{|c|c|c|c|c|}
\hline & \multicolumn{2}{|c|}{ Proteasome-mediated degradation } & \multirow{2}{*}{ Ubiquitin-mediated activation } & \multirow{2}{*}{ Deubiquitination } \\
\hline & Cellular E3 & Viral proteins & & \\
\hline IRF1 & CHIP, MDM2 & & cIAP2 & \\
\hline IRF2 & MDM2 & & & \\
\hline IRF3 & $\begin{array}{l}\text { PIN1 } \\
\text { TRIM21 } \\
\text { c-Cb1 } \\
\text { RBCK1 } \\
\text { RAUL }\end{array}$ & $\begin{array}{c}\text { SVV }\left(3 C^{\text {pro }}\right) \\
\left.\text { CSFV/BVDV (N }{ }^{\text {pro }}\right) \\
\text { RVs (NSP1) } \\
\text { HSV-1 (ICP0) } \\
\text { HIV-1 (Vif,Vpr,Vpu) } \\
\text { VZV (ORF61) }\end{array}$ & LUBAC & PLP2 \\
\hline IRF4 & Cbls & & & USP4 \\
\hline IRF5 & TRIM21 & RVs (NSP1) & TRAF6 & \\
\hline IRF7 & $\begin{array}{l}\text { TRIM21 } \\
\text { RAUL }\end{array}$ & $\begin{array}{c}\left.\text { SVV (3C }{ }^{\text {pro }}\right) \\
\text { RVs (NSP1) } \\
\text { KSHV (RTA) } \\
\text { GCRV (VP56) }\end{array}$ & $\begin{array}{l}\text { TRAF6 } \\
\text { RIP }\end{array}$ & $\mathrm{A} 20$ \\
\hline IRF8 & Cbls & & TRIM21 & USP4 \\
\hline IRF9 & & $\begin{array}{c}\text { RVs (NSP1) } \\
\text { VZV/SVV (ORF63) }\end{array}$ & & \\
\hline
\end{tabular}

may provide host targets for both antiviral treatment and autoimmune therapy.

Compliance and ethics The author(s) declare that they have no conflict of interest.

Acknowledgements This work was supported by the National Key Research and Development Program of China (2018YFA0800503 and 2018YFD0500100), an excellent young scientist foundation of NSFC (31822017), Zhejiang Provincial Natural Science Foundation of China (LR19C080001), the National Natural Science Foundation of China (81572651 and 81771675), and the Fundamental Research Funds for the Central Universities.

\section{References}

Arnold, M.M. (2016). The rotavirus interferon antagonist NSP1: Many targets, many questions. J Virol 90, 5212-5215.

Balkhi, M.Y., Fitzgerald, K.A., and Pitha, P.M. (2008). Functional regulation of MyD88-activated interferon regulatory factor 5 by K63linked polyubiquitination. Mol Cell Biol 28, 7296-7308.

Barro, M., and Patton, J.T. (2005). Rotavirus nonstructural protein 1 subverts innate immune response by inducing degradation of IFN regulatory factor 3. Proc Natl Acad Sci USA 102, 4114-4119.

Barro, M., and Patton, J.T. (2007). Rotavirus NSP1 inhibits expression of type I interferon by antagonizing the function of interferon regulatory factors IRF3, IRF5, and IRF7. J Virol 81, 4473-4481.

Bauhofer, O., Summerfield, A., Sakoda, Y., Tratschin, J.D., Hofmann, M. A., and Ruggli, N. (2007). Classical swine fever virus Npro interacts with interferon regulatory factor 3 and induces its proteasomal degradation. J Virol 81, 3087-3096.

Bhoj, V.G., and Chen, Z.J. (2009). Ubiquitylation in innate and adaptive immunity. Nature 458, 430-437.

Cao, C., An, R., Yu, Y.Y., Dai, H.Y., Qu, Z.H., Gao, M.C., and Wang, J.W. (2019). BICP0 negatively regulates TRAF6-mediated NF- $\mathrm{KB}$ and interferon activation by promoting K48-linked polyubiquitination of TRAF6. Front Microbiol 10, 3040.

Chattopadhyay, S., Kuzmanovic, T., Zhang, Y., Wetzel, J.L., and Sen, G.C.
(2016). Ubiquitination of the transcription factor IRF-3 activates RIPA, the apoptotic pathway that protects mice from viral pathogenesis. Immunity 44, 1151-1161.

Chattopadhyay, S., and Sen, G.C. (2017). RIG-I-like receptor-induced IRF3 mediated pathway of apoptosis (RIPA): a new antiviral pathway. Protein Cell 8, 165-168.

Chen, Y., Wu, Y., Yang, H., Li, X., Jie, M., Hu, C., Wu, Y., Yang, S., and Yang, Y. (2018). Prolyl isomerase Pin1: a promoter of cancer and a target for therapy. Cell Death Dis 9, 883 .

Chen, Z., Rijnbrand, R., Jangra, R.K., Devaraj, S.G., Qu, L., Ma, Y., Lemon, S.M., and Li, K. (2007). Ubiquitination and proteasomal degradation of interferon regulatory factor-3 induced by Npro from a cytopathic bovine viral diarrhea virus. Virology 366, 277-292.

Chen, Z.J., and Sun, L.J. (2009). Nonproteolytic functions of ubiquitin in cell signaling. Mol Cell 33, 275-286.

Deribe, Y.L., Pawson, T., and Dikic, I. (2010). Post-translational modifications in signal integration. Nat Struct Mol Biol 17, 666-672.

Doehle, B.P., Chang, K., Fleming, L., McNevin, J., Hladik, F., McElrath, M.J., and Gale Jr., M. (2012). Vpu-deficient HIV strains stimulate innate immune signaling responses in target cells. J Virol 86, 84998506.

Doehle, B.P., Hladik, F., McNevin, J.P., McElrath, M.J., and Gale Jr., M. (2009). Human immunodeficiency virus type 1 mediates global disruption of innate antiviral signaling and immune defenses within infected cells. J Virol 83, 10395-10405.

Dove, K.K., and Klevit, R.E. (2017). RING-between-RING E3 ligases: emerging themes amid the variations. J Mol Biol 429, 3363-3375.

Gao, B., Wang, Y., Xu, W., Li, S., Li, Q., and Xiong, S. (2013). Inhibition of histone deacetylase activity suppresses IFN- $\gamma$ induction of tripartite motif 22 via CHIP-mediated proteasomal degradation of IRF-1. J Immunol 191, 464-471.

Gatti, M., Pinato, S., Maiolica, A., Rocchio, F., Prato, M.G., Aebersold, R., and Penengo, L. (2015). RNF168 promotes noncanonical K27 ubiquitination to signal DNA damage. Cell Rep 10, 226-238.

Guo, Z., Xu, P., Ge, S., Zhang, C., Zheng, X., Xu, J., Liu, Z., Li, B., and Ge, S. (2017). Ubiquitin specific peptidase 4 stabilizes interferon regulatory factor protein and promotes its function to facilitate interleukin-4 expression in T helper type 2 cells. Int J Mol Med 40, 979-986.

Harada, H., Kitagawa, M., Tanaka, N., Yamamoto, H., Harada, K., Ishihara, M., and Taniguchi, T. (1993). Anti-oncogenic and oncogenic potentials 
of interferon regulatory factors-1 and -2. Science 259, 971-974.

Harikumar, K.B., Yester, J.W., Surace, M.J., Oyeniran, C., Price, M.M., Huang, W.C., Hait, N.C., Allegood, J.C., Yamada, A., Kong, X., et al. (2014). K63-linked polyubiquitination of transcription factor IRF1 is essential for IL-1-induced production of chemokines CXCL10 and CCL5. Nat Immunol 15, 231-238.

Heaton, S.M., Borg, N.A., and Dixit, V.M. (2016). Ubiquitin in the activation and attenuation of innate antiviral immunity. J Exp Med 213, $1-13$.

Higgs, R., and Jefferies, C.A. (2008). Targeting IRFs by ubiquitination: regulating antiviral responses. Biochem Soc Trans 36, 453-458.

Higgs, R., Lazzari, E., Wynne, C., Ni Gabhann, J., Espinosa, A., WahrenHerlenius, M., and Jefferies, C.A. (2010). Self protection from anti-viral responses - Ro52 promotes degradation of the transcription factor IRF7 downstream of the viral Toll-Like receptors. PLoS One 5, e11776.

Higgs, R., Gabhann, J.N., Larbi, N.B., Breen, E.P., Fitzgerald, K.A., and Jefferies, C.A. (2008). The E3 ubiquitin ligase Ro52 negatively regulates IFN- $\beta$ production post-pathogen recognition by polyubiquitin-mediated degradation of IRF3. J Immunol 181, 17801786.

Huang, H., Jeon, M., Liao, L., Yang, C., Elly, C., Yates Iii, J.R., and Liu, Y. C. (2010). K33-linked polyubiquitination of T cell receptor- $\zeta$ regulates proteolysis-independent $\mathrm{T}$ cell signaling. Immunity 33, 60-70.

Huye, L.E., Ning, S., Kelliher, M., and Pagano, J.S. (2007). Interferon regulatory factor 7 is activated by a viral oncoprotein through RIPdependent ubiquitination. Mol Cell Biol 27, 2910-2918.

Ikeda, F., and Dikic, I. (2008). Atypical ubiquitin chains: new molecular signals. 'Protein Modifications: Beyond the Usual Suspects' review series. EMBO Rep 9, 536-542.

Jefferies, C.A. (2019). Regulating IRFs in IFN driven disease. Front Immunol 10, 325.

Jin, S.M., Lazarou, M., Wang, C., Kane, L.A., Narendra, D.P., and Youle, R.J. (2010). Mitochondrial membrane potential regulates PINK1 import and proteolytic destabilization by PARL. J Cell Biol 191, 933-942.

Karin, M., and Ben-Neriah, Y. (2000). Phosphorylation meets ubiquitination: the control of NF- $\mathrm{KB}$ activity. Annu Rev Immunol 18, 621-663.

Komander, D., and Rape, M. (2012). The ubiquitin code. Annu Rev Biochem 81, 203-229.

Kong, H.J., Anderson, D.E., Lee, C.H., Jang, M.K., Tamura, T., Tailor, P., Cho, H.K., Cheong, J.H., Xiong, H., Morse Iii, H.C., et al. (2007). Cutting edge: autoantigen Ro52 is an interferon inducible E3 ligase that ubiquitinates IRF-8 and enhances cytokine expression in macrophages. J Immunol 179, 26-30.

Landré, V., Pion, E., Narayan, V., Xirodimas, D.P., and Ball, K.L. (2013). DNA-binding regulates site-specific ubiquitination of IRF-1. Biochem J 449, 707-717.

Langer, S., Hammer, C., Hopfensperger, K., Klein, L., Hotter, D., De Jesus, P.D., Herbert, K.M., Pache, L., Smith, N., van der Merwe, J.A., et al. (2019). HIV-1 Vpu is a potent transcriptional suppressor of NF-кBelicited antiviral immune responses. eLife 8, e41930.

Lazear, H.M., Lancaster, A., Wilkins, C., Suthar, M.S., Huang, A., Vick, S. C., Clepper, L., Thackray, L., Brassil, M.M., Virgin, H.W., et al. (2013). IRF-3, IRF-5, and IRF-7 coordinately regulate the type I IFN response in myeloid dendritic cells downstream of MAVS signaling. PLoS Pathog 9, e1003118.

Lazzari, E., Korczeniewska, J., Ni Gabhann, J., Smith, S., Barnes, B.J., and Jefferies, C.A. (2014). TRIpartite motif 21 (TRIM21) differentially regulates the stability of interferon regulatory factor 5 (IRF5) isoforms. PLoS One 9, e103609.

Li, X., Gadzinsky, A., Gong, L., Tong, H., Calderon, V., Li, Y., Kitamura, D., Klein, U., Langdon, W.Y., Hou, F., et al. (2018). Cbl ubiquitin ligases control B cell exit from the germinal-center reaction. Immunity 48, 530-541.e6.

Lin, R., Nie, J., Ren, J., Liang, R., Li, D., Wang, P., Gao, C., Zhuo, C., Yang, C., and Li, B. (2017). USP4 interacts and positively regulates IRF8 function via K48-linked deubiquitination in regulatory $\mathrm{T}$ cells.
FEBS Lett 591, 1677-1686.

Liu, J., Qian, C., and Cao, X. (2016). Post-translational modification control of innate immunity. Immunity 45, 15-30.

Marsili, G., Perrotti, E., Remoli, A.L., Acchioni, C., Sgarbanti, M., and Battistini, A. (2016). IFN regulatory factors and antiviral innate immunity: how viruses can get better. J Interferon Cytokine Res 36, 414-432.

Moll, U.M., and Petrenko, O. (2003). The MDM2-p53 interaction. Mol Cancer Res 1, 1001-1008.

Mukhopadhyay, D., and Riezman, H. (2007). Proteasome-independent functions of ubiquitin in endocytosis and signaling. Science 315, 201205.

Murata, S., Minami, Y., Minami, M., Chiba, T., and Tanaka, K. (2001). CHIP is a chaperone-dependent E3 ligase that ubiquitylates unfolded protein. EMBO Rep 2, 1133-1138.

Narayan, V., Pion, E., Landré, V., Müller, P., and Ball, K.L. (2011). Docking-dependent ubiquitination of the interferon regulatory factor-1 tumor suppressor protein by the ubiquitin ligase CHIP. J Biol Chem 286, 607-619.

Nehyba, J., Hrdlickova, R., and Bose, H.R. (2009). Dynamic evolution of immune system regulators: the history of the interferon regulatory factor family. Mol Biol Evol 26, 2539-2550.

Nijman, S.M.B., Luna-Vargas, M.P.A., Velds, A., Brummelkamp, T.R., Dirac, A.M.G., Sixma, T.K., and Bernards, R. (2005). A genomic and functional inventory of deubiquitinating enzymes. Cell 123, 773-786.

Ning, S., Campos, A.D., Darnay, B.G., Bentz, G.L., and Pagano, J.S. (2008). TRAF6 and the three C-terminal lysine sites on IRF7 are required for its ubiquitination-mediated activation by the tumor necrosis factor receptor family member latent membrane protein 1 . Mol Cell Biol 28, 6536-6546.

Ning, S., Pagano, J.S., and Barber, G.N. (2011). IRF7: activation, regulation, modification and function. Genes Immun 12, 399-414.

Ning, S., and Pagano, J.S. (2010). The A20 deubiquitinase activity negatively regulates LMP1 activation of IRF7. J Virol 84, 61306138.

Okumura, A., Alce, T., Lubyova, B., Ezelle, H., Strebel, K., and Pitha, P.M. (2008). HIV-1 accessory proteins VPR and Vif modulate antiviral response by targeting IRF-3 for degradation. Virology 373, 85-97.

Pettersson, S., Kelleher, M., Pion, E., Wallace, M., and Ball, K.L. (2009). Role of Mdm2 acid domain interactions in recognition and ubiquitination of the transcription factor IRF-2. Biochem J 418, 575585.

Pion, E., Narayan, V., Eckert, M., and Ball, K.L. (2009). Role of the IRF-1 enhancer domain in signalling polyubiquitination and degradation. Cell Signal 21, 1479-1487.

Randow, F., and Lehner, P.J. (2009). Viral avoidance and exploitation of the ubiquitin system. Nat Cell Biol 11, 527-534.

Remoli, A.L., Marsili, G., Perrotti, E., Acchioni, C., Sgarbanti, M., Borsetti, A., Hiscott, J., and Battistini, A. (2016). HIV-1 tat recruits HDM2 E3 ligase to target IRF-1 for ubiquitination and proteasomal degradation. mBio 7, e01528.

Rhodes, D.A., Ihrke, G., Reinicke, A.T., Malcherek, G., Towey, M., Isenberg, D.A., and Trowsdale, J. (2002). The 52000 MW Ro/SS-A autoantigen in Sjogren's syndrome/systemic lupus erythematosus (Ro52) is an interferon-gamma inducible tripartite motif protein associated with membrane proximal structures. Immunology 106, 246-256.

Richardson, R.J., Dixon, J., Malhotra, S., Hardman, M.J., Knowles, L., Boot-Handford, R.P., Shore, P., Whitmarsh, A., and Dixon, M.J. (2006). Irf6 is a key determinant of the keratinocyte proliferation-differentiation switch. Nat Genet 38, 1329-1334.

Saira, K., Zhou, Y., and Jones, C. (2007). The infected cell protein 0 encoded by bovine herpesvirus 1 (bICP0) induces degradation of interferon response factor 3 and, consequently, inhibits beta interferon promoter activity. J Virol 81, 3077-3086.

Saira, K., Zhou, Y., and Jones, C. (2009). The infected cell protein 0 encoded by bovine herpesvirus 1 (bICP0) associates with interferon 
regulatory factor 7 and consequently inhibits beta interferon promoter activity. J Virol 83, 3977-3981.

Saitoh, T., Tun-Kyi, A., Ryo, A., Yamamoto, M., Finn, G., Fujita, T., Akira, S., Yamamoto, N., Lu, K.P., and Yamaoka, S. (2006). Negative regulation of interferon-regulatory factor 3-dependent innate antiviral response by the prolyl isomerase Pin1. Nat Immunol 7, 598-605.

Savitsky, D., Tamura, T., Yanai, H., and Taniguchi, T. (2010). Regulation of immunity and oncogenesis by the IRF transcription factor family. Cancer Immunol Immunother 59, 489-510.

Schneider, W.M., Chevillotte, M.D., and Rice, C.M. (2014). Interferonstimulated genes: a complex web of host defenses. Annu Rev Immunol $32,513-545$.

Stacey, K.B., Breen, E., and Jefferies, C.A. (2012). Tyrosine phosphorylation of the E3 ubiquitin ligase TRIM21 positively regulates interaction with IRF3 and hence TRIM21 activity. PLoS ONE 7, e34041.

Swaminathan, G., and Tsygankov, A.Y. (2006). The Cbl family proteins: ring leaders in regulation of cell signaling. J Cell Physiol 209, 21-43.

Takaoka, A., Hayakawa, S., Yanai, H., Stoiber, D., Negishi, H., Kikuchi, H., Sasaki, S., Imai, K., Shibue, T., Honda, K., et al. (2003). Integration of interferon- $\alpha / \beta$ signalling to $p 53$ responses in tumour suppression and antiviral defence. Nature 424, 516-523.

Tamura, T., Yanai, H., Savitsky, D., and Taniguchi, T. (2008). The IRF family transcription factors in immunity and oncogenesis. Annu Rev Immunol 26, 535-584.

Thien, C.B.F., and Langdon, W.Y. (2005). c-Cbl and Cbl-b ubiquitin ligases: substrate diversity and the negative regulation of signalling responses. Biochem J 391, 153-166.

Tian, Y., Zhang, Y., Zhong, B., Wang, Y.Y., Diao, F.C., Wang, R.P., Zhang, M., Chen, D.Y., Zhai, Z.H., and Shu, H.B. (2007). RBCK1 negatively regulates tumor necrosis factor- and interleukin-1-triggered $\mathrm{NF}-\mathrm{\kappa B}$ activation by targeting TAB $2 / 3$ for degradation. J Biol Chem 282, 16776-16782.

Tokunaga, F., and Iwai, K. (2012). LUBAC, a novel ubiquitin ligase for linear ubiquitination, is crucial for inflammation and immune responses. Microbes Infection 14, 563-572.

Tokunaga, F., Sakata, S., Saeki, Y., Satomi, Y., Kirisako, T., Kamei, K., Nakagawa, T., Kato, M., Murata, S., Yamaoka, S., et al. (2009). Involvement of linear polyubiquitylation of NEMO in NF- $\mathrm{KB}$ activation. Nat Cell Biol 11, 123-132.

Tsuchida, T., Kawai, T., and Akira, S. (2009). Inhibition of IRF3-dependent antiviral responses by cellular and viral proteins. Cell Res 19, 3-4.

Verweij, M.C., Wellish, M., Whitmer, T., Malouli, D., Lapel, M., Jonjic, S., Haas, J.G., DeFilippis, V.R., Mahalingam, R., and Fruh, K. (2015). Varicella viruses inhibit interferon-stimulated JAK-STAT signaling through multiple mechanisms. PLoS Pathog 11, e1004901.

Wang, Q., Liu, X., Cui, Y., Tang, Y., Chen, W., Li, S., Yu, H., Pan, Y., and Wang, C. (2014). The E3 ubiquitin ligase AMFR and INSIG1 bridge the activation of TBK1 kinase by modifying the adaptor STING. Immunity 41, 919-933.

Welchman, R.L., Gordon, C., and Mayer, R.J. (2005). Ubiquitin and ubiquitin-like proteins as multifunctional signals. Nat Rev Mol Cell Biol 6, 599-609.

Wu-Baer, F., Lagrazon, K., Yuan, W., and Baer, R. (2003). The BRCA1/
BARD1 heterodimer assembles polyubiquitin chains through an unconventional linkage involving lysine residue K6 of ubiquitin. J Biol Chem 278, 34743-34746.

Xiong, H., Li, H., Kong, H.J., Chen, Y., Zhao, J., Xiong, S., Huang, B., Gu, H., Mayer, L., Ozato, K., et al. (2005). Ubiquitin-dependent degradation of interferon regulatory factor- 8 mediated by $\mathrm{Cbl}$ down-regulates interleukin-12 expression. J Biol Chem 280, 23531-23539.

Xue, Q., Liu, H., Zhu, Z., Yang, F., Ma, L., Cai, X., Xue, Q., and Zheng, H. (2018a). Seneca Valley Virus 3Cpro abrogates the IRF3- and IRF7mediated innate immune response by degrading IRF3 and IRF7. Virology 518, 1-7.

Xue, Q., Liu, H., Zhu, Z., Yang, F., Xue, Q., Cai, X., Liu, X., and Zheng, H. (2018b). Seneca Valley Virus 3C protease negatively regulates the type I interferon pathway by acting as a viral deubiquitinase. Antiviral Res $160,183-189$.

Yanai, H., Negishi, H., and Taniguchi, T. (2012). The IRF family of transcription factors. Oncoimmunology 1, 1376-1386.

Yang, K., Shi, H.X., Liu, X.Y., Shan, Y.F., Wei, B., Chen, S., and Wang, C. (2009). TRIM21 is essential to sustain IFN regulatory factor 3 activation during antiviral response. J Immunol 182, 3782-3792.

Yang, M., Chen, T., Li, X., Yu, Z., Tang, S., Wang, C., Gu, Y., Liu, Y., Xu, S., Li, W., et al. (2015). K33-linked polyubiquitination of Zap70 by Nrdp1 controls $\mathrm{CD}^{+}$T cell activation. Nat Immunol 16, 1253-1262.

Young, J.A., Sermwittayawong, D., Kim, H.J., Nandu, S., An, N., Erdjument-Bromage, H., Tempst, P., Coscoy, L., and Winoto, A. (2011). Fas-associated death domain (FADD) and the E3 ubiquitinprotein ligase TRIM21 interact to negatively regulate virus-induced interferon production. J Biol Chem 286, 6521-6531.

Yu, Y., and Hayward, G.S. (2010). The ubiquitin E3 ligase RAUL negatively regulates type I interferon through ubiquitination of the transcription factors IRF7 and IRF3. Immunity 33, 863-877.

Yu, Y., Wang, S.E., and Hayward, G.S. (2005). The KSHV immediate-early transcription factor RTA encodes ubiquitin E3 ligase activity that targets IRF7 for proteosome-mediated degradation. Immunity 22, 59-70.

Zhang, C., Lu, L.F., Li, Z.C., Zhou, X.Y., Zhou, Y., Chen, D.D., Li, S., and Zhang, Y.A. (2020). Grass carp reovirus VP56 represses interferon production by degrading phosphorylated IRF7. Fish Shellfish Immunol 99, 99-106.

Zhang, M., Tian, Y., Wang, R.P., Gao, D., Zhang, Y., Diao, F.C., Chen, D. Y., Zhai, Z.H., and Shu, H.B. (2008). Negative feedback regulation of cellular antiviral signaling by RBCK1-mediated degradation of IRF3. Cell Res 18, 1096-1104.

Zhao, X., Zhu, H., Yu, J., Li, H., Ge, J., and Chen, W. (2016). c-Cblmediated ubiquitination of IRF3 negatively regulates IFN- $\beta$ production and cellular antiviral response. Cell Signal 28, 1683-1693.

Zheng, D., Chen, G., Guo, B., Cheng, G., and Tang, H. (2008). PLP2, a potent deubiquitinase from murine hepatitis virus, strongly inhibits cellular type I interferon production. Cell Res 18, 1105-1113.

Zheng, N., and Shabek, N. (2017). Ubiquitin ligases: structure, function, and regulation. Annu Rev Biochem 86, 129-157.

Zhu, H., Zheng, C., Xing, J., Wang, S., Li, S., Lin, R., and Mossman, K.L. (2011). Varicella-zoster virus immediate-early protein ORF61 abrogates the IRF3-mediated innate immune response through degradation of activated IRF3. J Virol 85, 11079-11089. 